\title{
Clinical implications of microvascular CT scan signs in COVID-19 patients requiring invasive mechanical ventilation
}

\author{
Giorgia Dalpiaz ${ }^{1}$ - Lorenzo Gamberini ${ }^{2}$ - Aldo Carnevale ${ }^{3}$. Savino Spadaro ${ }^{4}$. Carlo Alberto Mazzoli ${ }^{2}$ \\ Sara Piciucchi ${ }^{5} \cdot$ Davide Allegri $^{6} \cdot$ Chiara Capozzi $^{7} \cdot$ Ersenad Neziri $^{8} \cdot$ Maurizio Bartolucci $^{9} \cdot$ Francesco Muratore $^{10}$. \\ Francesca Coppola ${ }^{11}$. Antonio Poerio ${ }^{12}$. Emanuela Giampalma ${ }^{13}$. Luca Baldini $^{14}$. Tommaso Tonetti $^{15}$. \\ lacopo Cappellini ${ }^{16}$. Davide Colombo ${ }^{17,18}$. Gianluca Zani ${ }^{19} \cdot$ Lorenzo Mellini $^{20}$. Vanni Agnoletti ${ }^{21}$. \\ Federica Damiani ${ }^{22}$. Giovanni Gordini ${ }^{2}$ - Cristiana Laici ${ }^{23}$. Giuliano Gola ${ }^{24}$. Antonella Potalivo ${ }^{25}$. \\ Jonathan Montomoli ${ }^{26}$. Vito Marco Ranieri ${ }^{15}$. Emanuele Russo ${ }^{21}$. Stefania Taddei ${ }^{27}$. Carlo Alberto Volta ${ }^{4}$. \\ Gaetano Scaramuzzo ${ }^{4}$
}

Received: 23 July 2021 / Accepted: 21 December 2021 / Published online: 16 January 2022

(C) Italian Society of Medical Radiology 2022

\begin{abstract}
Purpose COVID-19-related acute respiratory distress syndrome (ARDS) is characterized by the presence of signs of microvascular involvement at the CT scan, such as the vascular tree in bud (TIB) and the vascular enlargement pattern (VEP). Recent evidence suggests that TIB could be associated with an increased duration of invasive mechanical ventilation (IMV) and intensive care unit (ICU) stay. The primary objective of this study was to evaluate whether microvascular involvement signs could have a prognostic significance concerning liberation from IMV.

Material and methods All the COVID-19 patients requiring IMV admitted to 16 Italian ICUs and having a lung CT scan recorded within 3 days from intubation were enrolled in this secondary analysis. Radiologic, clinical and biochemical data were collected.

Results A total of 139 patients affected by COVID-19 related ARDS were enrolled. After grouping based on TIB or VEP detection, we found no differences in terms of duration of IMV and mortality. Extension of VEP and TIB was significantly correlated with ground-glass opacities (GGOs) and crazy paving pattern extension. A parenchymal extent over 50\% of GGO and crazy paving pattern was more frequently observed among non-survivors, while a VEP and TIB extent involving 3 or more lobes was significantly more frequent in non-responders to prone positioning.

Conclusions The presence of early CT scan signs of microvascular involvement in COVID-19 patients does not appear to be associated with differences in duration of IMV and mortality. However, patients with a high extension of VEP and TIB may have a reduced oxygenation response to prone positioning.
\end{abstract}

Trial Registration: NCT04411459

Keywords Novel coronavirus disease 2019 · Acute respiratory distress syndrome $\cdot$ Pulmonary perfusion · Thoracic imaging $\cdot$ Mechanical ventilation

$\begin{array}{ll}\text { Abbreviations } \\ \text { ARDS } & \text { Acute respiratory distress syndrome } \\ \text { ICU } & \text { Intensive care unit } \\ \text { CT } & \text { Computed tomography } \\ \text { VEP } & \text { Vascular enlargement pattern } \\ \text { TIB } & \text { Tree in bud } \\ \text { GGOs } & \text { Ground-glass opacities }\end{array}$

Lorenzo Gamberini

lorenzo.gamberini86@gmail.com

Extended author information available on the last page of the article

$\begin{array}{ll}\text { NIV } & \text { Non-invasive ventilation } \\ \text { IMV } & \text { Invasive mechanical ventilation } \\ \text { VFD } & \text { Ventilator-free days } \\ \text { SAPS II } & \text { Simplified acute physiology score } \\ \text { SOFA } & \text { Sequential organ failure assessment } \\ \text { LDH } & \text { Lactate dehydrogenase } \\ \text { CRP } & \text { C-reactive protein } \\ \text { PP } & \text { Prone positioning } \\ \text { IQR } & \text { Interquartile range }\end{array}$

NIV Non-invasive ventilation

IMV Invasive mechanical ventilation

VFD Ventilator-free days

SAPS II Simplified acute physiology score

SOFA Sequential organ failure assessment

LDH Lactate dehydrogenase

CRP C-reactive protein

$\begin{array}{ll}\mathrm{PP} & \text { Prone positioning } \\ \mathrm{IQR} & \text { Interquartile range }\end{array}$ 


\section{Introduction}

COVID-19 is a multisystem syndrome due to cellular infection via the ACE 2 receptors by SARS-CoV-2. This syndrome most commonly involves the respiratory system, with $15 \%$ of cases showing severe respiratory failure and 5\% requiring intensive care unit (ICU) admission for acute respiratory distress syndrome (ARDS) or multiorgan failure [1].

Thrombotic complications are frequently observed in COVID-19 patients [2] and, especially for COVID19 related ARDS, the observed incidence of pulmonary embolism (PE) and alveolar-capillary microthrombosis are significantly higher than for other ARDS causes [3, 4].

A combination of conventional mechanisms of clot formation and immunothrombosis (particularly for smaller clots) seems to underlie these manifestations, and more recent insights about the interactions between SARS$\mathrm{CoV}-2$ and the vascular endothelium led to the definition of a COVID-19-associated coagulopathy.

This syndrome is characterized by mild thrombocytopenia, mildly prolonged prothrombin time, increased fibrinogen and raised D-dimer, all of which are more pronounced as disease severity increases $[5,6]$.

From the histopathological point of view, other than the classical signs of ARDS such as diffuse alveolar damage and hyaline membrane formation, some distinctive pulmonary microvascular abnormalities occur in COVID-19, including intravascular fibrin deposition, perivascular monocyte infiltration, angiogenesis and microthrombi formation [4].

Endothelial inflammation and microthrombosis can justify a series of CT scan abnormalities, such as vascular enlargement pattern (VEP) and vascular tree in bud (TIB), which have been increasingly described in COVID-19 CT scans $[7,8]$.

The VEP is characterized by dilatation of pulmonary vessels around and within the lung abnormalities on CT images [8,9], and different descriptive approaches for VEP were adopted in the available literature, such as vascular thickening [10], subsegmental vessels greater than $3 \mathrm{~mm}$ [11] or dilated small vessels in the lesion [12].

It appears to be related to the vasodilatation and damage characterized by endothelial injury and enhanced permeability due to the massive accumulation of pro-inflammatory factors in the lung [13]. An increased representation of the VEP is observed along with the increase in the extent of ground-glass opacities (GGOs) and with the clinical severity of COVID-19 symptoms [8].

On the other hand, TIB is the expression of pulmonary microthrombosis that enhances the peripheral pulmonary vessels on CT imaging, therefore producing the TIB pattern [14].
Vascular TIB identification was correlated with an increased duration of mechanical ventilation (MV) and length of hospital stay in a cohort of 39 critically ill invasively ventilated COVID-19 patients [7].

It is not clear if these radiological signs represent only an expression of the extension of the COVID-19-related lung inflammation or if they could have an independent role in determining the severity of the clinical manifestations and the duration of invasive mechanical ventilation (IMV). Moreover, no evidence exists that patients responding differently to some of the COVID-19 related ARDS treatments, such as prone positioning, have different patterns of vascular involvement [15].

Therefore, the primary objective of this study was to assess whether the presence of microvascular damage signs (VEP and TIB) in the chest CT scans acquired within 3 days before or after endotracheal intubation could be independently associated with prolonged IMV in critically ill COVID-19 patients.

Secondary objectives were to find eventual influences of the extension of these signs on the response to prone positioning and ICU outcome measures.

\section{Material and methods}

\section{Setting and ethics}

This is a secondary analysis of patients enrolled in a previous prospective study [16] initially conducted in 15 ICUs from Italian hospitals between February 22 and May 4, 2020. The data from another ICU obtained after the publication of the first paper were included in the present report.

The study was approved by the Institutional Review Board of the study coordinator centre (Maggiore Hospital, Bologna, Italy, approval number: 273/2020/OSS/AUSLBO) and by each institutional review committee of the participating hospitals. Informed consent was partially waived according to the approval of the local Ethics committee and analysis was conducted on anonymized individual data. The study was registered in ClinicalTrials.gov (NCT04411459).

\section{Outcome measures}

The primary outcome measure was the number of ventilatorfree days (VFDs) at day 28 (see below for the full definition) between two groups based on the presence/absence of vascular TIB [7] or VEP.

Secondary outcome measures were the differences in VFD at day $28, \mathrm{PaO}_{2} / \mathrm{FiO}_{2}$ ratio change after pronation and ICU mortality between groups of patients showing different extensions of the microvascular and parenchymal signs. In particular, the microvascular involvement of 3 or more lobes 
for VES and TIB and the parenchymal involvement of over $50 \%$ for the parenchymal signs were used to define groups of patients with extended radiologic involvement.

\section{Definitions}

Vascular TIB was defined as the presence of dilated peripheral subpleural pulmonary small vessels with a branching aspect resembling a budding tree $[7,17]$.

VEP was defined as an ectasia of segmental and subsegmental vessels with a diameter larger than expected for the point within the vascular tree, characterized by: (a) vessel diameter larger than that in adjacent portions of the nondiseased lung, (b) vessel diameter larger than that in comparable regions of the non-diseased contralateral lung, or (c) focal dilatation or non-tapering of vessels as they course towards the lung periphery [18].

Detailed images of TIB and VEP are available in Fig. 1.

For the other signs, the definitions were referred to the Glossary of Terms for Thoracic Imaging of the Fleischner Society [19].

Mechanical ventilation was considered invasive if delivered through an endotracheal tube or a tracheostomy cannula. The duration of IMV was defined as the time elapsed from intubation to successful extubation or successful permanent disconnection from mechanical ventilation for tracheostomized patients. This latter was considered effective if sustained $24 \mathrm{~h}$ per day. VFDs at 28 days were defined as 28 minus the number of days elapsed from the last successful extubation in intubated patients, whether or not NIV was used after extubation. VFDs were defined as zero in patients who died during the 28 days regardless of their extubation status. In tracheostomized patients, intermittent disconnections were not counted and VFD were defined as 28 minus the number of days from the last successful sustained disconnection from mechanical ventilation [20].

\section{Inclusion and exclusion criteria}

To be included in the present study, patients should be tested positive for SARS-CoV-2 infection (confirmed by real-time reverse transcription-polymerase chain reaction assays), older than 18 years, undergo IMV, fulfil the criteria for ARDS according to the Berlin definition [21], and have at least one CT scan available performed within three days before or after orotracheal intubation.

The single non-inclusion criterion was the use of noninvasive ventilation (NIV) during the entire clinical course.

\section{Sample size calculation}

The sample size was determined considering 5 VFD as a clinically important difference on a baseline mean of

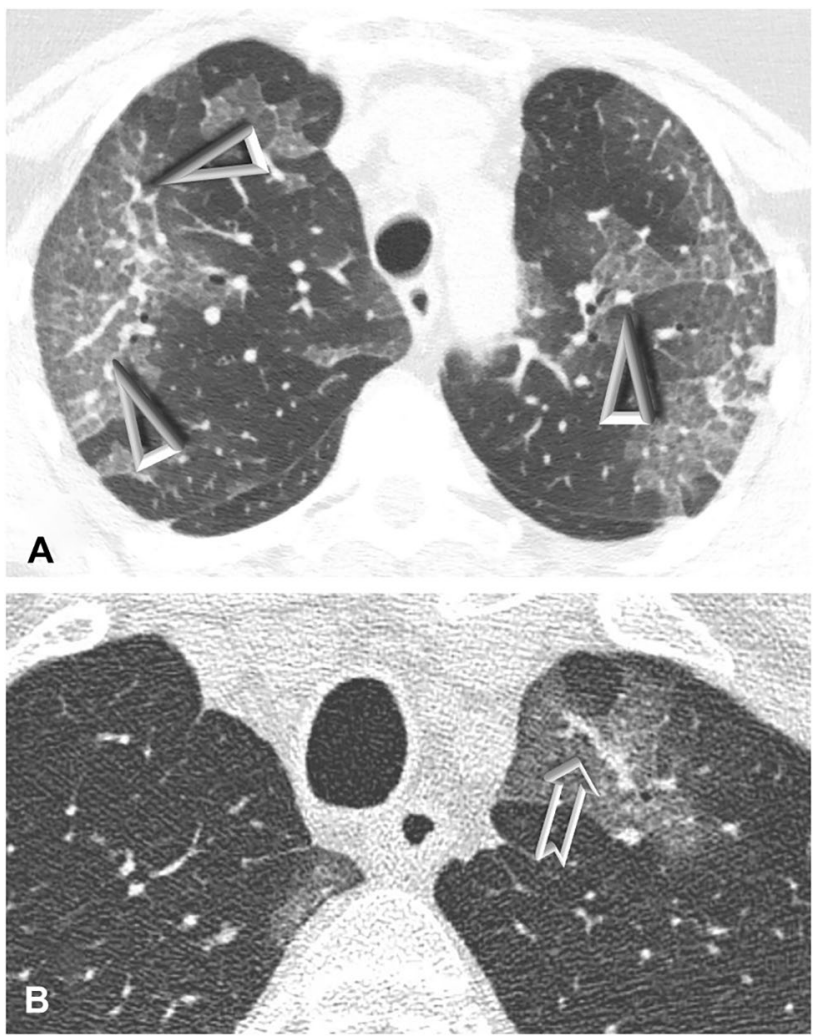

Fig. 1 CT scans showing vascular enlargement pattern (VEP) and vascular tree-in-bud (TIB) in two patients with COVID-19 pneumonia. Notes: A-Bilateral peripheral areas of ground-glass opacification and crazy paving in the upper lobes. Dilated segmental and subsegmental vessels (VEP_arrowheads) are visible inside these areas. $\mathrm{B}$ - Targeted image shows striking dilatation of peripheral subpleural vessel in upper left lobe with a branching aspect resembling a budding tree (arrow). Please note that vascular TIB is visible only within the area of ground-glass-opacity in this picture

$11 \pm 7.5$ VFD [16], and considering an $\alpha$-error of 0.05 and a $\beta$-error of 0.8 with a 1:1 ratio between the two groups, a sample size of at least 126 patients was required.

\section{Data collection}

Baseline data and patient's history were collected using an electronic case report form developed by YGHEA, CRO division of Ecol Studio SPA (Bologna Operational Headquarters, Bologna, Italy) and hosted by Actide Nubilaria (Novara, Italy). Collected data included demographic data, underlying comorbidities, laboratory data, chest radiological reports, respiratory parameters before the intubation and during the first five days of IMV (static compliance $-C_{R S}$, $\mathrm{PaO}_{2} / \mathrm{FiO}_{2}$ ratio), and clinical outcomes.

Radiological data recorded consisted in the presence, extent and distribution of VEP, TIB and peripheral micronodules, scored on the basis of the number of lobes involved; GGO, crazy paving and consolidations, evaluated 
for percentage of lung involvement on a scale of 0 to 4 as follows: $0=0 \%$ involvement, $1=$ less than $25 \%, 2=25 \%$ to less than $50 \%, 3=50 \%$ to less than $75 \%, 4=75 \%$ or greater involvement. The concomitant presence of pleural effusion and pulmonary artery dilatation (diameter greater than $29 \mathrm{~mm}$ ) were also recorded.

Concerning the extent of the lesions, VEP, TIB and peripheral nodules were considered extended if they involved 3 or more lobes, while GGO, crazy paving and consolidations were considered extended if they involved more than $50 \%$ of the pulmonary parenchyma.

Concerning pronation-related data, the $\mathrm{PaO}_{2} / \mathrm{FiO}_{2}$ was recorded within $3 \mathrm{~h}$ before prone positioning (T1) and from 1 to $3 \mathrm{~h}$ after re-supination (T2), all data were referred to the first pronation and both measurements were taken, therefore, in the supine position, and the duration of the PP session was recorded. $\mathrm{A} \mathrm{PaO}_{2} / \mathrm{FiO}_{2}$ increase of at least $49 \%$ was used as a cut-off value to identify responders to pronation [15].

\section{CT scan acquisition}

Chest CT studies were interpreted by the means of a consensus assessment from two radiologists with at least 5 years of experience for each centre. If consensus was not reached, a senior radiologist was consulted to address the final decision.

CT scan acquisitions were performed using different scanners in different institutions: GE VCT Lightspeed 64, GE VCT Lightspeed 16 (GE Healthcare, Buckinghamshire, UK), Philips CT Ingenuity 64, Philips Brilliance 64 (Philips Medical Systems, Best, The Netherlands), Toshiba Aquilion S16 TSX-101A and Toshiba Aquilion S32 TSX101A (Toshiba Medical Systems Europe, Zoetermeer, The Netherlands).

The scans included non-contrast acquisition and were obtained in the supine position from lung apices to bases at full-suspended inspiration, with $\leq 1.25$-mm section thicknesses, using standard acquisition parameters adjusted to patients' biometrics (100-120 effective mAs and 120-140 $\mathrm{kVp}$ ). Images were reconstructed using both sharp kernels and visualized at window settings optimized for lung parenchyma (window width, $1500 \mathrm{HU}$; window level, -500 HU), and medium-soft kernels with soft-tissue window settings (window width, $300 \mathrm{HU}$; window level, $40 \mathrm{HU}$ ). Maximum intensity projection (MIP) images were used to facilitate the recognition of the TIB and VEP.

\section{Statistical analysis}

Data were analysed using Stata/CI 16 (StataCorp LLC, Texas, USA) and GraphPad Prism version 8.4.3 for Windows (GraphPad Software, San Diego, California, USA).

Continuous variables were tested for normality with the Shapiro-Wilk test and were expressed as mean and standard deviation (SD) or median and interquartile range [IQR] based on their distribution. Comparisons between continuous variables were made with the Student's t-test or the Mann-Whitney U test when appropriate. Categorical variables were expressed as numbers and percentages and compared using the Chi-square test or Fisher's exact test when needed.

The log-rank test was used to compare the cumulative incidences of liberation from mechanical ventilation up to day 28 between the groups of patients with and without TIB and VEP. Kaplan-Meier curves were also built for the same groups.

Finally, bivariate Spearman correlation coefficients were calculated taking into account the extension of the different radiological signs.

\section{Results}

During the study period, 632 patients were assessed for eligibility. After excluding those who underwent exclusively NIV or with missing data, the original study enrolled a total of 470 patients. Of these, 139 had a CT scan performed within \pm 3 days from orotracheal intubation and were therefore included in the final analysis; supplement Fig. 1 shows the flow of the patients throughout the study.

Globally, the TIB sign was observed in 75 out of the 139 patients (54\%), while the VEP in 108 out of 139 (77.7\%); Table 1 shows the main demographic and clinical variables of the patients included, while Table 2 describes their clinical course.

The TIB sign was associated with a significantly lower BMI and a small, but statistically significant, delay from hospital to ICU admission and orotracheal intubation; these differences were not observed concerning the presence of the VEP. Patients' demographics were not significantly different with regard to age, comorbidities, clinical severity at ICU admission (SAPS II and SOFA score) as well as for the clinical severity of COVID-19-related ARDS in terms of worst $\mathrm{PaO}_{2} / \mathrm{FiO}_{2}$ ratio and lowest respiratory system compliance $\left(C_{\mathrm{RS}}\right)$ observed during the first five days of IMV for both the TIB and VEP comparisons.

Supplement Table 1 shows the initial laboratory and ventilatory data. The nearest D-dimer concentration from the CT scan as well as the peak concentration of IL- 6 and LDH was not significantly different between the two groups; however, the prevalence of patients with $\mathrm{LDH}>250 \mathrm{U} / \mathrm{L}$ [22] was significantly higher in the VEP group ( $99 \%$ vs $85.7 \%$, $p=0.008$ ). There were no significant differences in terms of responders to prone positioning either in the TIB (58.7\% vs $55.8 \%, p=0.832)$ and VEP groups $(50.8 \%$ vs $73.1 \%$, $p=0.063)$. 
Table 1 Demographics, comorbidities, orotracheal intubation timing and ICU admission severity

\begin{tabular}{|c|c|c|c|c|c|c|c|}
\hline & All $(n=139)$ & TIB $(n=75)$ & No TIB $(n=64)$ & $p$ & $\operatorname{VEP}(n=108)$ & No VEP $(n=31)$ & $p$ \\
\hline \multicolumn{8}{|l|}{ Demographics and comorbidities } \\
\hline Age-yr (IQR) & $66(57-74)$ & $67(59-74)$ & $64(57-74)$ & 0.404 & $68(59-74)$ & $63(56-70)$ & 0.115 \\
\hline Sex-male-no $(\%)$ & $104(74.8 \%)$ & $54(72 \%)$ & $50(78.1 \%)$ & 0.407 & $80(74.1 \%)$ & $24(77.4 \%)$ & 0.705 \\
\hline BMI—median (IQR) & $28(25-32)$ & $28(25-31)$ & $29(26-33)$ & $\mathbf{0 . 0 3 3}$ & $28(25-31)$ & $28(25-33)$ & 0.838 \\
\hline Hypertension-no (\%) & $86(61.9 \%)$ & $41(54.7 \%)$ & $45(70.3 \%)$ & 0.058 & $65(60.2 \%)$ & $21(67.7 \%)$ & 0.445 \\
\hline Chronic ischemic heart disease-no (\%) & $12(8.6 \%)$ & $5(6.7 \%)$ & $7(10.9 \%)$ & 0.372 & $8(7.4 \%)$ & $4(12.9 \%)$ & \\
\hline Chronic kidney disease (CKD) & & & & $0.567^{*}$ & & & $0.790^{*}$ \\
\hline CKD—not in dialysis—no (\%) & $7(5.0 \%)$ & $2(3.1 \%)$ & $5(6.7 \%)$ & & $6(5.6 \%)$ & $1(3.2 \%)$ & \\
\hline $\mathrm{CKD}$ - patients in dialysis—no (\%) & $3(2.2 \%)$ & $2(2.7 \%)$ & $1(1.6 \%)$ & & $2(1.9 \%)$ & $1(3.2 \%)$ & \\
\hline COPD—no $(\%)$ & $8(5.8 \%)$ & $6(8.0 \%)$ & $2(3.1 \%)$ & $0.267^{*}$ & $6(5.6 \%)$ & $2(6.5 \%)$ & $0.852^{*}$ \\
\hline $\begin{array}{l}\text { COPD—home oxygen therapy/CPAP—no } \\
(\%)\end{array}$ & $1(0.7 \%)$ & $0(0 \%)$ & $1(1.6 \%)$ & & $1(0.9 \%)$ & $0(0 \%)$ & \\
\hline Diabetes-no (\%) & $36(25.9 \%)$ & $18(24 \%)$ & $18(28.1 \%)$ & 0.580 & $30(27.8 \%)$ & $6(19.4 \%)$ & 0.345 \\
\hline \multicolumn{8}{|l|}{ ICU admission and orotracheal intubation } \\
\hline $\begin{array}{l}\text { Time from hospital admission to ICU } \\
\text { admission- }-\mathrm{d} \text { (IQR) }\end{array}$ & $1(0-2)$ & $1(1-3)$ & $1(0-2)$ & 0.004 & $1(0-3)$ & $1(0-2)$ & 0.089 \\
\hline $\begin{array}{l}\text { Time from hospital admission to intuba- } \\
\text { tion-d (IQR) }\end{array}$ & $1(0-3)$ & $2(1-3)$ & $1(0-2)$ & 0.002 & $1(0-3)$ & $1(0-2)$ & 0.108 \\
\hline SAPS II score & $35(29-43)$ & $35(29-43)$ & $35(30-46)$ & 0.488 & $35(29-43)$ & $35(29-46)$ & 0.757 \\
\hline SOFA score at ICU admission & $5(3-7)$ & $5(3-7)$ & $5(3-7)$ & 0.790 & $5(3-7)$ & $5(3-7)$ & 0.927 \\
\hline
\end{tabular}

$B M I$ body mass index, $C K D$ chronic kidney disease, $C O P D$ chronic obstructive pulmonary disease, $I C U$ intensive care unit, SAPS simplified acute physiology score, SOFA sequential organ failure assessment, IQR interquartile range

Significant $p$ values are reported in bold. *Refers to the $\mathrm{p}$ value of the Chi-square test for the whole contingency table for the variable taken into account

Table 3 shows the extent and distribution of the main radiologic signs found in the $\mathrm{CT}$ scans included, while Fig. 2 represents the correlation matrix of the radiologic variables taken into account. In most cases, both TIB and VEP involved two or three lobes; the extents of the two signs were significantly intercorrelated and also correlated with the percentage of pulmonary parenchyma affected by GGO. The magnitude of the association was weak to moderate (rho coefficients range: $0.32-0.35, p<0.01$ ), TIB extent was also significantly correlated with the extent of peripheral micronodules (rho: $0.26, p<0.01$ ).

Vascular TIB was mostly observed within the GGO areas, while VEP was observed inside the GGO areas in $61.1 \%$ and both inside and outside the GGO areas in $34.3 \%$ of the cases.

Interestingly, pulmonary artery enlargement was significantly more frequently observed in patients with TIB sign, while this observation was not confirmed for VEP.

Duration of mechanical ventilation was similar between patients with and without TIB in CT scan (15 [8-23] versus 16 [8-24], respectively, $p=0.297)$ as well as $28 \mathrm{~d}-\mathrm{VFD}(0$ $[0-15]$ vs 0 [0-12], $p=0.979)$. Similar results were found when dividing the two groups based on the presence of the VEP. Figure 3 shows the Kaplan-Meier graph for the cumulative incidence of liberation from mechanical ventilation within the first 28 days after orotracheal intubation based on the presence of the TIB sign or the VEP and the relative logrank tests; no differences were found in the two functions.

Finally, ICU cardiovascular complications and the need for renal replacement therapy were not significantly different in either of the two grouping modalities (VEP and TIB presence), as well as ICU mortality (Table 2).

Figure 4 shows the distribution of extended radiological signs among different subgroups of patients.

Extended VEP and TIB signs were significantly more frequent in poor responders to pronation, while consolidations were more frequent in responders. Ground glass and crazy paving pattern extension over $50 \%$ of pulmonary parenchyma were significantly more frequent in non-survivors. No differences were observed among the radiologic signs concerning weaning from MV within day 28 and $\mathrm{PaO}_{2} / \mathrm{FiO}_{2}$ ratio before orotracheal intubation.

\section{Discussion}

Microvascular involvement is a key determinant of COVID19 syndrome, and microvascular signs such as VEP and TIB are part of the typical radiological aspects of this syndrome and have an established role in its diagnosis $[8,12,23]$. 
Table 2 Ventilatory data, adjunctive therapies, ICU stay and outcomes

\begin{tabular}{|c|c|c|c|c|c|c|c|}
\hline & All $(n=139)$ & TIB $(n=75)$ & No TIB $(n=64)$ & $p$ & $\operatorname{VEP}(n=108)$ & No VEP $(n=31)$ & $p$ \\
\hline \multicolumn{8}{|c|}{ Mechanical ventilation-first 5 days and adjunctive therapies } \\
\hline $\begin{array}{l}\text { Lowest } \mathrm{PaO}_{2} / \mathrm{FiO}_{2} \text { ratio in supine posi- } \\
\text { tion-(IQR) }\end{array}$ & $99(72-132)$ & $102(70-133)$ & $90(75-129)$ & 0.546 & $100(72-133)$ & $90(65-115)$ & 0.360 \\
\hline $\begin{array}{l}\text { Lowest } \mathrm{PaO}_{2} / \mathrm{FiO}_{2} \text { ratio in supine posi- } \\
\text { tion-no }(\%)\end{array}$ & & & & $0.098^{*}$ & & & $0.434^{*}$ \\
\hline $200-300-$ no $(\%)$ & $6(4.3 \%)$ & $4(5.3 \%)$ & $2(3.1 \%)$ & & $5(4.6 \%)$ & $1(3.2 \%)$ & \\
\hline $100-200-$ no $(\%)$ & $62(44.6 \%)$ & $39(52.0 \%)$ & $23(35.9 \%)$ & & $51(47.2 \%)$ & $11(35.5 \%)$ & \\
\hline$<100-$ no $(\%)$ & $71(51.1 \%)$ & $32(42.7 \%)$ & $39(60.9 \%)$ & & $52(48.1 \%)$ & $19(61.3 \%)$ & \\
\hline Lowest static $\mathrm{C}_{\mathrm{RS}}-\mathrm{mL} / \mathrm{cmH} 2 \mathrm{O}$ (IQR) & $40(32-47)$ & $39(30-47)$ & $40(35-48)$ & 0.186 & $40(32-47)$ & $40(30-48)$ & 0.950 \\
\hline $\mathrm{C}_{\mathrm{RS}}<40 \mathrm{~mL} / \mathrm{cmH} 2 \mathrm{O}-$ no $(\%)$ & $65(46.8 \%)$ & $38(50.7 \%)$ & $27(42.2 \%)$ & 0.318 & $49(45.4 \%)$ & $16(51.6 \%)$ & 0.539 \\
\hline $\begin{array}{l}\text { Neuromuscular blocking agents continuous } \\
\text { infusion-no }(\%)\end{array}$ & $124(89.1 \%)$ & $67(89.3 \%)$ & $57(89.1 \%)$ & 0.959 & $97(89.8 \%)$ & $27(87.1 \%)$ & 0.667 \\
\hline Any thromboprophylaxis-no (\%) & $139(100 \%)$ & $75(100 \%)$ & $64(100 \%)$ & 1.000 & $108(100 \%)$ & $31(100 \%)$ & 1.000 \\
\hline Enoxaparin therapy-no (\%) & $184(89.2 \%)$ & $69(92 \%)$ & $55(85.9 \%)$ & 0.251 & $97(89.8 \%)$ & $27(87.1 \%)$ & 0.667 \\
\hline Enoxaparin daily dose-mg (IQR) & $60(40-80)$ & $60(40-80)$ & $60(40-80)$ & 0.393 & $60(40-80)$ & $60(40-80)$ & 0.782 \\
\hline Intravenous corticosteroids-no $(\%)$ & $96(69.1 \%)$ & $56(74.7 \%)$ & $40(62.5 \%)$ & 0.122 & $77(71.3 \%)$ & $19(61.3 \%)$ & 0.288 \\
\hline Antiplatelet therapy-no (\%) & $44(31.7 \%)$ & $24(32 \%)$ & $20(31.3 \%)$ & 0.925 & $33(30.6 \%)$ & $11(35.5 \%)$ & 0.603 \\
\hline \multicolumn{8}{|l|}{ ICU stay and outcomes } \\
\hline Tracheotomy-no (\%) & $89(64.0 \%)$ & $44(58.7 \%)$ & $45(70.3 \%)$ & 0.154 & $72(66.7 \%)$ & $17(54.8 \%)$ & 0.226 \\
\hline Prone positioning-no (\%) & $83(59.7 \%)$ & $43(57.3 \%)$ & $40(62.5 \%)$ & 0.536 & $65(60.2 \%)$ & $18(58.1 \%)$ & 0.832 \\
\hline Cardiovascular complications-no (\%) & $35(25.2 \%)$ & $21(28.0 \%)$ & $14(21.9 \%)$ & 0.407 & $28(25.9 \%)$ & $7(22.6 \%)$ & 0.705 \\
\hline Need for RRT—no $(\%)$ & $31(22.3 \%)$ & $14(18.7 \%)$ & $17(26.6 \%)$ & 0.265 & $26(24.1 \%)$ & $5(16.1 \%)$ & 0.349 \\
\hline Duration of MV—days (IQR) & $15(9-24)$ & $15(8-23)$ & $16(8-24)$ & 0.297 & $15(9-24)$ & $16(9-23)$ & 0.652 \\
\hline VFD-28 days-days (IQR) & $0(0-14)$ & $0(0-15)$ & $0(0-12)$ & 0.979 & $0(0-15)$ & $0(0-12)$ & 0.980 \\
\hline ICU length of stay-days (IQR) & $18(12-32)$ & $19(13-31)$ & $17(9-33)$ & 0.558 & $19(13-32)$ & $17(9-33)$ & 0.652 \\
\hline ICU mortality-no (\%) & $57(41.0 \%)$ & $32(42.7 \%)$ & $25(39.1 \%)$ & 0.667 & $46(42.6 \%)$ & $11(35.5 \%)$ & 0.478 \\
\hline
\end{tabular}

$C_{R S}$ respiratory system compliance, $I Q R$ interquartile range

Significant $p$ values are reported in bold. *Refers to the $p$ value of the Chi-square test for the whole contingency table for the variable taken into account

However, the clinical implications of these signs are still poorly investigated.

The main findings of this study were: (a) the evidence of VEP or TIB on CT scans performed within 3 days from orotracheal intubation was not associated with different clinical courses in terms of duration of MV or mortality; (b) no differences were either found about the median concentration of biomarkers related to disease severity and thrombosis; however, VEP was significantly associated with a slightly higher prevalence of $\mathrm{LDH}>250 \mathrm{U} / \mathrm{L}$ and TIB was associated with an increased prevalence of pulmonary artery enlargement; (c) the extent of microvascular signs was significantly correlated with that of GGO, peripheral micronodules and crazy paving pattern, while no correlation was found with the extent of consolidations; (d) the involvement of 3 or more lobes by VEP and TIB was more frequent among nonresponders to prone positioning.

Recent literature found a significant association between the evidence of VEP or TIB in CT scan imaging and disease severity in terms of symptoms and biomarkers, impaired coagulation and an increase in the duration of MV and hospital stay [7, 23].

Among COVID-19 patients showing vascular changes in CT scan, a significantly increased proportion of elevated C-reactive protein and LDH was observed [23], and the elevation of these biomarkers was significantly associated with ICU admission and mortality in the available literature $[1,24]$.

Our results showed no differences in median values of LDH; however, VEP was associated with a significantly increased proportion of patients with peak LDH values above $250 \mathrm{U} / \mathrm{L}$ during the first 7 days of hospitalization [22].

On the other hand, TIB was associated with a significantly increased prevalence of pulmonary artery dilatation, a radiologic sign recently recognized as a predictor of clinical severity and mortality in COVID-19 patients [25, 26].

However, despite the higher prevalence of negative prognostic factors in the VEP and TIB populations, we were not able to demonstrate any significant difference in terms of liberation from mechanical ventilation and mortality. 
Table 3 Main radiologic findings

Vascular signs and peripheral nodules-number of lobes involved

\begin{tabular}{|c|c|c|c|c|c|c|}
\hline & Present & 1 & 2 & 3 & 4 & 5 \\
\hline Vascular tree in bud-no (\%) & $75(54 \%)$ & $7(9.3 \%)$ & $26(34.7 \%)$ & $23(30.7 \%)$ & $9(12 \%)$ & $10(13.3 \%)$ \\
\hline Vascular enlargement pattern-no (\%) & $108(77.7 \%)$ & $3(2.8 \%)$ & $24(22.2 \%)$ & $26(24.1 \%)$ & $17(15.7 \%)$ & $38(35.2 \%)$ \\
\hline Peripheral nodules-no (\%) & $24(17.3 \%)$ & $2(8.3 \%)$ & $10(41.7 \%)$ & $9(37.5 \%)$ & $3(12.5 \%)$ & $0(0 \%)$ \\
\hline \multicolumn{7}{|c|}{ Vascular signs and peripheral nodules_-distribution and localization } \\
\hline & \multicolumn{3}{|l|}{ Distribution } & \multicolumn{3}{|l|}{ Localization } \\
\hline & Anterior & Intermediate & Posterior & Inside GGO & Outside GGO & Both \\
\hline Vascular tree in bud $(n=75)-$ no $(\%)$ & $43(57.3 \%)$ & $59(78.7 \%)$ & $38(50.7 \%)$ & $63(84 \%)$ & $0(0 \%)$ & $12(16 \%)$ \\
\hline $\begin{array}{l}\text { Vascular enlargement pattern } \\
(n=108) \text { - no }(\%)\end{array}$ & $74(68.5 \%)$ & $89(82.4 \%)$ & $80(74.1 \%)$ & $66(61.1 \%)$ & $5(4.6 \%)$ & $37(34.3 \%)$ \\
\hline Peripheral nodules $(n=24)-$ no $(\%)$ & $12(50 \%)$ & $20(83.3 \%)$ & $9(37.5 \%)$ & $16(66.7 \%)$ & $2(8.3 \%)$ & $6(25.0 \%)$ \\
\hline
\end{tabular}

Ground glass opacities, crazy paving and consolidations-visual extension and distribution

\begin{tabular}{|c|c|c|c|c|c|c|}
\hline & Present & $1-25 \%$ & $26-50 \%$ & $51-75 \%$ & $76-100 \%$ & Main distribution (A/P/D) \\
\hline Ground glass opacities-no (\%) & $131(94.2 \%)$ & $49(37.4 \%)$ & $35(26.7 \%)$ & $31(23.7 \%)$ & $16(12.2 \%)$ & $\begin{array}{l}\text { A - } 21(16 \%) \\
\text { P - } 4(3.1 \%) \\
\text { D - } 106(80.9 \%)\end{array}$ \\
\hline Crazy paving-no (\%) & $71(51.1 \%)$ & $37(52.1 \%)$ & $15(21.1 \%)$ & $15(21.1 \%)$ & $4(5.6 \%)$ & $\begin{array}{l}\text { A - } 14(19.7 \%) \\
\text { P - } 9(12.7 \%) \\
\text { D - } 48(67.6 \%)\end{array}$ \\
\hline Consolidations-no (\%) & $120(86.3 \%)$ & $76(63.3 \%)$ & $35(29.2 \%)$ & $5(4.2 \%)$ & $4(3.3 \%)$ & $\begin{array}{l}\text { A - } 5(4.2 \%) \\
\text { P - } 89(74.2 \%) \\
\text { D - } 26(21.7 \%)\end{array}$ \\
\hline
\end{tabular}

Other radiologic findings

\begin{tabular}{|c|c|c|c|c|c|c|}
\hline & TIB $(n=75)$ & No TIB $(n=64)$ & $p$ & $\operatorname{VEP}(n=108)$ & No VEP $(n=31)$ & $p$ \\
\hline Pleural effusion- $n(\%)$ & & & $0.467^{*}$ & & & $0.260^{*}$ \\
\hline Monolateral & $6(8 \%)$ & $2(3.1 \%)$ & & $5(4.6 \%)$ & $3(9.7 \%)$ & \\
\hline Bilateral & $7(9.3 \%)$ & $6(9.4 \%)$ & & $12(11.1 \%)$ & $1(3.2 \%)$ & \\
\hline $\begin{array}{l}\text { Pulmonary artery enlargement } \\
\text { (>29 mm }-n(\%)\end{array}$ & $52(69.3 \%)$ & $23(35.9 \%)$ & $<0.001$ & $60(55.6 \%)$ & $15(48.4 \%)$ & 0.480 \\
\hline
\end{tabular}

Main distribution: $A$ anterior, $P$ posterior, $D$ diffuse

Significant $p$ values are reported in bold. *Refers to the $p$ value of the Chi-square test for the whole contingency table for the variable taken into account

Moreover, the number of lobes involved by VEP and TIB was correlated with a higher extent of the GGO (Fig. 2), which has been already associated with worse outcomes in several studies [27-29], and this aspect was also confirmed in our study where we found a significantly higher prevalence of extended GGO among non-survivors (Fig. 4).

The pulmonary epithelial and endothelial damage due to SARS-CoV-2, the subsequent release of inflammatory cytokines and the proinflammatory/anti-inflammatory unbalance seem to be a shared mechanism between the development of the microvascular abnormalities, oedema and exudate formation [30], of which GGOs are the radiologic expression $[23,31]$.
Therefore, based on our results, VEP and TIB extent could be a partial epiphenomenon of GGO extent, without a prognostic significance in terms of duration of IMV or mortality in critically ill patients.

Interestingly, extended microvascular signs were significantly more frequent among non-responders to prone positioning, this aspect is in line with our previous research [15], which found that the $\mathrm{PaO}_{2} / \mathrm{FiO}_{2}$ increase observed after prone positioning in sustained responders could not be fully explained by differences in driving pressure. Possibly, disease phenotypes showing an extensive microvascular involvement could blunt the oxygenation response to prone positioning, while phenotypes showing extended 


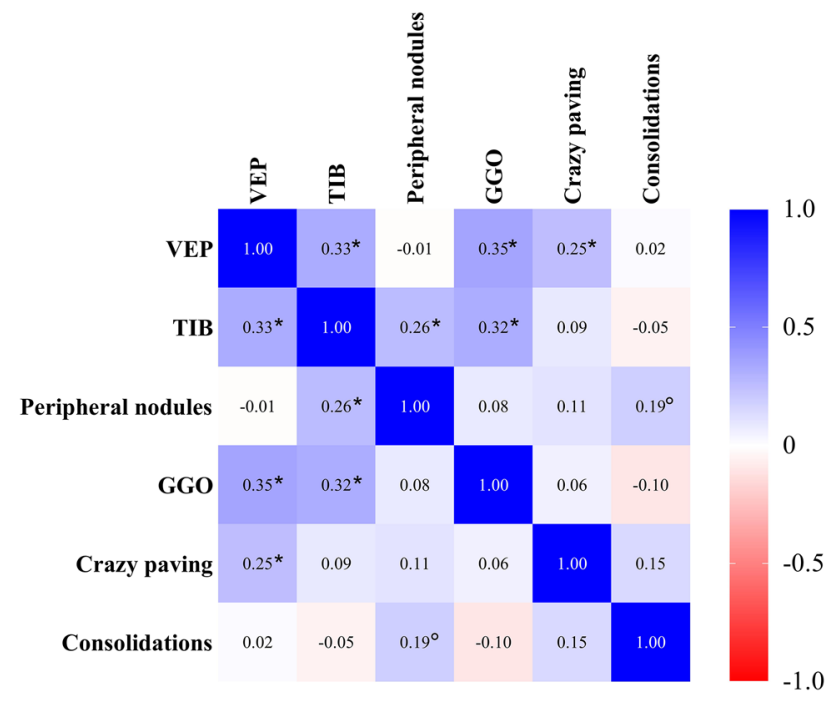

Fig. 2 Spearman heatmap regarding the extension of the radiologic signs explored. Abbreviations: VEP — vascular enlargement pattern; TIB - tree in bud sign; GGO-ground glass opacities. Notes-the values reported in the cells refer to the rho coefficient for the bivariate correlation of the two variables; $p<0.05 ;{ }^{*} p<0.01$

consolidations rely more on parenchymal recruitment and ventilation/perfusion ratio redistribution for their oxygenation response.

Unfortunately, prone positioning was performed in only $83(59.7 \%)$ of the patients enrolled in the present study; therefore, we were limited in performing subgroup analyses concerning the anteroposterior distribution of vascular lesions or extent of consolidations. The mixed population of patients treated and not treated with prone positioning in this study could have also played a role concerning the observed liberation from MV.

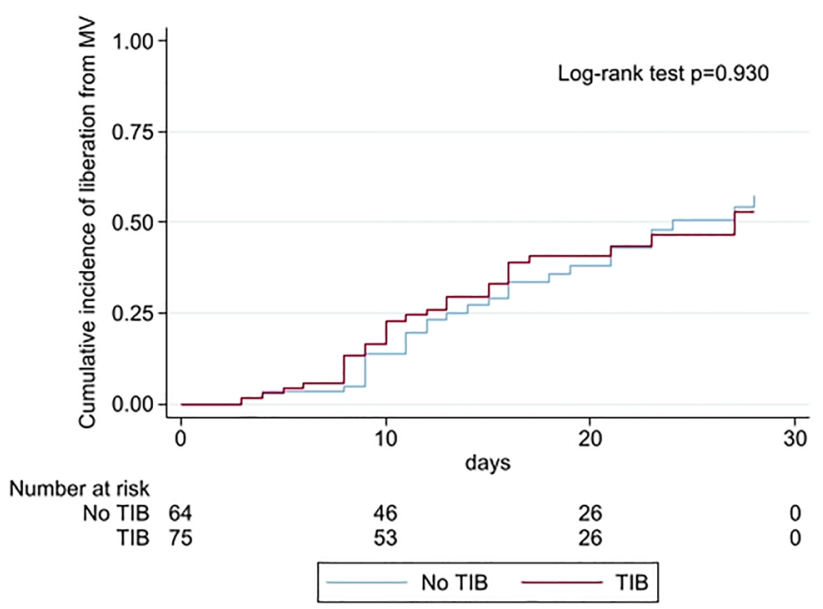

Our results could have also been partially influenced by the selection criteria adopted for patients' enrolment because, since this study focused exclusively on critically ill COVID-19 patients, a higher prevalence of negative prognostic risk factors is expected, and the relative eventual prognostic value of vascular signs could have been smoothened in favour of other factors that notoriously play a role in critically ill patients such as the number of concomitant organ failures [16, 32], hospital-acquired superinfections [33, 34] and other ICU complications [2, 35].

Another limitation of this study is related to the different experimental treatments that were adopted for patients undergoing IMV during the study period and that could have partially influenced our results.

Moreover, we could not get more detailed lung perfusion images due to the lack of systematic acquisition of pulmonary CT angiograms in the patients included and the unavailability of dual-energy computed tomography (DECT) machines in the centres participating in this study, differently from previous works [7]. Finally, it should be noted that consolidations imply the increase in parenchymal attenuation that obscures the margins of vessels, and this may have influenced the prevalence of microvascular signs in patients demonstrating more consolidated lung areas.

Future research should further define if vascular signs are only a radiologic epiphenomenon of a common shared pathogenetic mechanism with GGO or have an independent clinical meaning and their eventual relation with COVID-19-related coagulopathy.

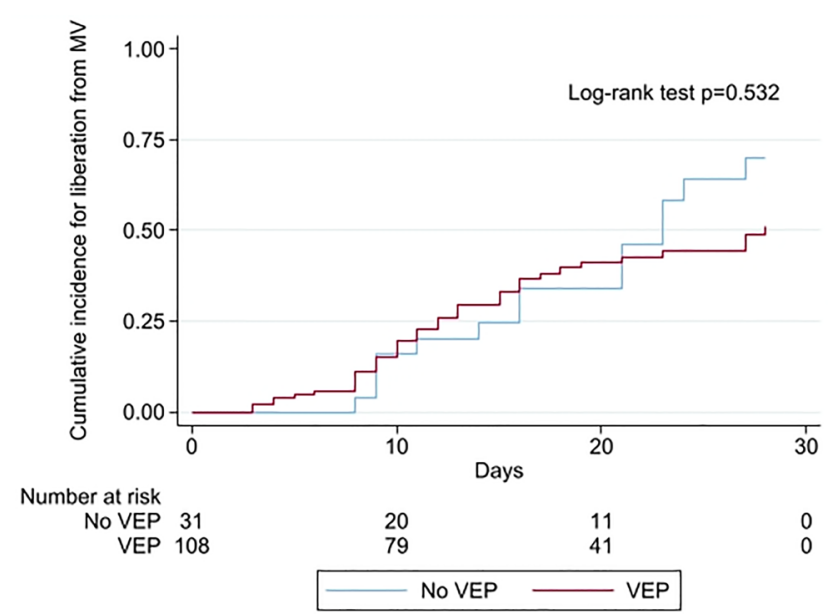

Fig. 3 Cumulative incidence functions of liberation from mechanical ventilation. Abbreviations: TIB — tree in bud; VEP-vascular enlargement pattern; MV-mechanical ventilation 


\section{Prone positioning response}

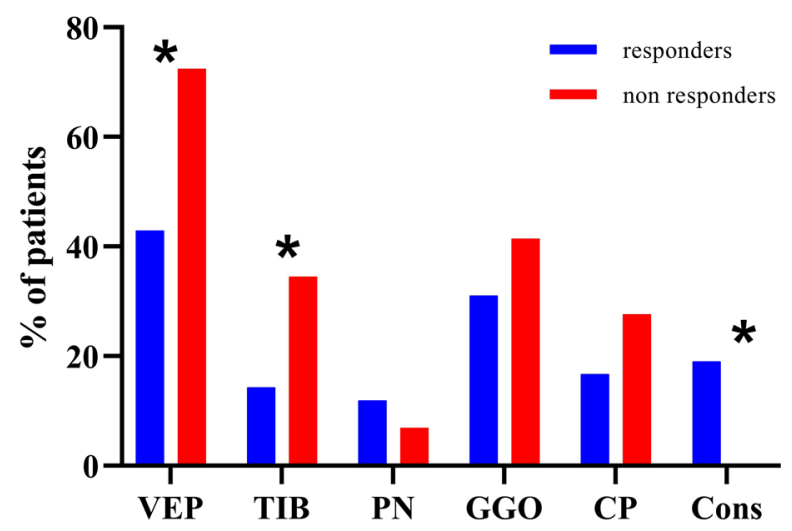

Outcome

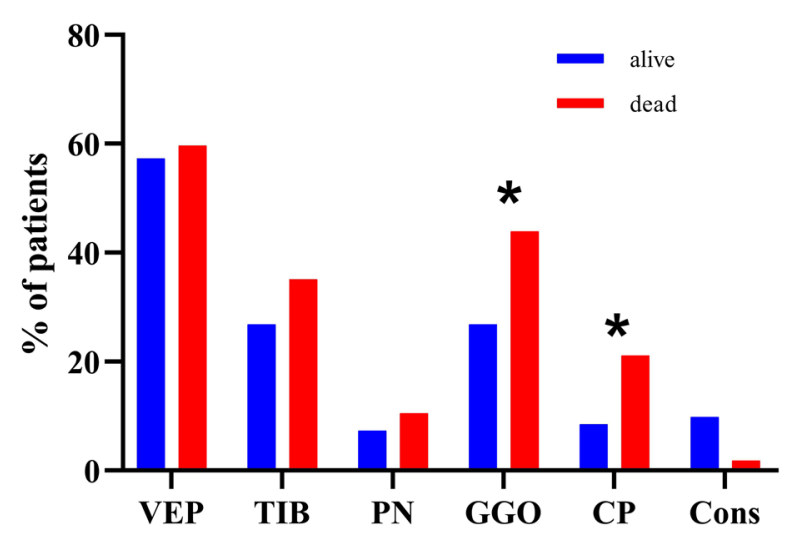

Fig. 4 Prevalence of extended radiologic signs in different contexts. Abbreviations: VEP-vascular enlargement pattern; TIB-tree in bud; PN—peripheral nodules; GGO_-ground glass opacities; $\mathrm{CP}$ crazy paving; Cons-consolidations. Notes: Graphs show the preva-

\section{Conclusions}

Vascular TIB and VEP are frequently observed in the CT scans of critically ill COVID-19 patients requiring IMV. Their extent is significantly correlated with GGO and crazy paving pattern areas. Despite previous studies suggesting a potential prognostic role of these signs concerning the duration of IMV and survival, and the detection of a higher prevalence of prognostic factors associated with prolonged ventilation in our population, we were not able to confirm this hypothesis.

Patients not responding to prone positioning had a higher extent of microvascular involvement signs. More focused studies on the potential relation between vascular signs and oxygenation response to prone positioning could better clarify if these signs could help in choosing which patients may benefit from prone positioning.

\section{$\mathrm{PaO}_{2} / \mathrm{FiO}_{2}$ before intubation}

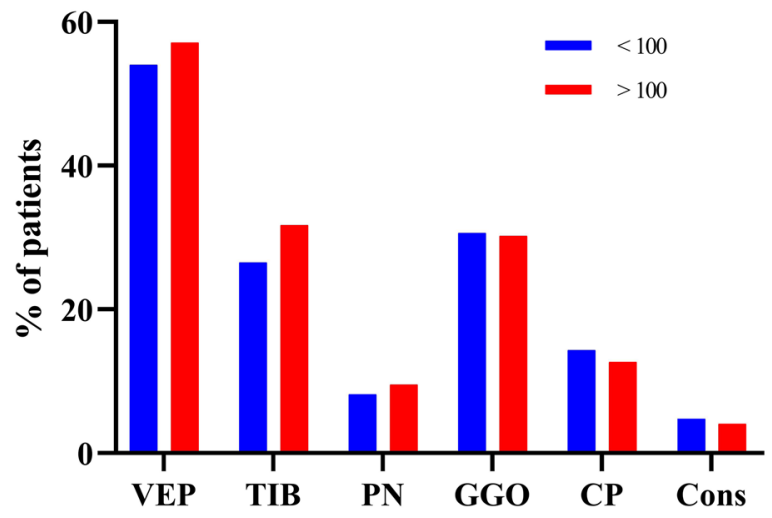

\section{Weaning from MV at 28 days}

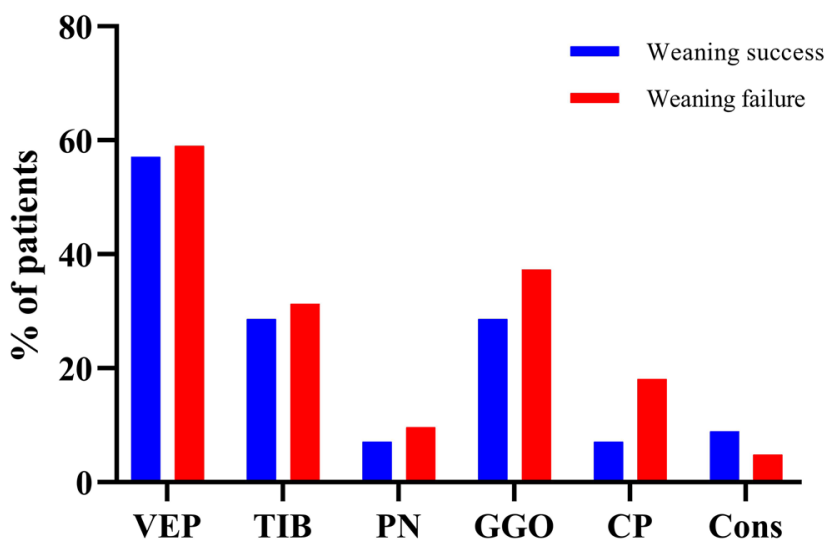

lence of VEP, TIB and PN involving 3 or more lobes and GGO, $\mathrm{CP}$ and Cons extended for over the $50 \%$ of pulmonary parenchyma. $* p<0.05$ at Chi-square test

Supplementary Information The online version contains supplementary material available at https://doi.org/10.1007/s11547-021-01444-7.

Acknowledgements ICU-RER COVID-19 Collaboration - List of collaborators (to be indexed and searchable into PubMed). ICU collaborators Maggiore Hospital Carlo Alberto Pizzardi, Bologna, IT: Marco Tartaglione, Valentina Chiarini, Virginia Buldini, Carlo Coniglio, Federico Moro, Federico Semeraro, Daniele Fecarotti. Bellaria Hospital, Bologna, IT: Nicola Cilloni, Lorenzo Giuntoli, Angela Bellocchio, Emanuele Matteo. Sant'Orsola-Malpighi University Hospital, Bologna, IT: Giacinto Pizzilli, Antonio Siniscalchi, Chiara Natalia Tartivita, Francesco Matteo, Andrea Castelli, Irene Cavalli. Imola Hospital, Bologna, IT: Annalisa Marchio, Igor Bacchilega. Infermi Hospital, Rimini, IT: Laura Bernabè, Francesca Facondini, Sonia Guarino, Luca Morini. M. Bufalini Hospital, Cesena, IT: Luca Bissoni, Lorenzo Viola, Emiliano Gamberini. SS. Trinità Hospital, ASL Novara, IT: Federico Crimaldi Alessia Guzzo. Bentivoglio Hospital, Bentivoglio, IT: Anna Malfatto, Angelina Adduci, Silvia Pareschi. University Hospital of Modena, Modena, IT: Gabriele Melegari, Jessica Maccieri, Elisabetta Bertellini, Andrea Marudi. Azienda Ospedaliera SS. Antonio e Biagio e Cesare Arrigo, Alessandria, IT: Fabrizio Racca, Mirco Leo. University of Ferrara, Azienda Ospedaliero-Universitaria S. Anna, Cona, 
Ferrara, IT: Marco Verri, Giulia Falò, Elisabetta Marangoni, Irene Ottaviani. Santa Maria delle Croci Hospital, Ravenna, IT: Maurizio Fusari, Giulia Felloni, Federico Domenico Baccarini. Morgagni-Pierantoni Hospital, Forlì, IT: Marina Terzitta, Stefano Maitan, Maria Concetta Lanza. Azienda USL Toscana Centro, Prato, IT: Filippo Becherucci, Guglielmo Consales, Lorenzo Tutino, Angelo Senzi. Radiology Collaborators (to be indexed and searchable into PubMed). Maggiore Hospital Carlo Alberto Pizzardi, Bologna, IT: Michele Imbriani, Paolo Orlandi. Sant'Orsola-Malpighi University Hospital, Bologna, IT: Rita Golfieri, Federica Ciccarese. Infermi Hospital, Rimini, IT: Fabio Ferrari. M. Bufalini Hospital, Cesena, IT: Martina Mughetti. SS. Trinità Hospital, ASL Novara, IT: Loredana Franchini. Bentivoglio Hospital, Bentivoglio, IT: Marco Miceli. Santa Maria delle Croci Hospital, Ravenna, IT: Maria Teresa Minguzzi

Authors contributions GD, LG, GS, AC, SS, SP, CC and CAM conceptualized and designed the work. EN, MB, FM, FC, AP, EG, LB, TT, IC, DC, GZ, LM, VA, FD, CL, GG, AP, VMR, GG, JM, ST and ER acquired and interpreted the data. DA, LG and GS performed the statistical analysis. LG, AC, GS and CAM drafted the article. GD, GS and CAV substantively revised the article. The ICU-RER COVID-19 collaboration was involved in data collection (see acknowledgments). All the authors have approved the submitted version and have agreed both to be personally accountable for the author's own contributions and to ensure that questions related to the accuracy or integrity of any part of the work, even ones in which the author was not personally involved.

Funding This research did not receive any specific grant from funding agencies in the public, commercial or not-for-profit sectors.

Data availability The dataset related to this publication is available at https://doi.org/10.17632/cmctw928j4.1.

\section{Declarations}

Conflict of interest The authors have nothing to disclose.

Ethics approval and consent to participate The study was approved by the Institutional Review Board (IRB) of the study coordinator centre (Maggiore Hospital, Bologna, Italy, Approval Number: 273/2020/OSS/ AUSLBO) and by each institutional review committee of the participating hospitals. Informed consent was waived for unconscious patients while it was acquired for conscious patients or after liberation from mechanical ventilation or at the time of follow-up. The researchers analysed anonymized individual data.

\section{References}

1. Wu Z, McGoogan JM (2020) Characteristics of and important lessons from the coronavirus disease 2019 (COVID-19) outbreak in China: summary of a report of 72314 cases from the chinese center for disease control and prevention. JAMA J Am Med Assoc 323:1239-1242

2. Lodigiani C, Iapichino G, Carenzo L et al (2020) Venous and arterial thromboembolic complications in COVID-19 patients admitted to an academic hospital in Milan, Italy. Thromb Res 191:9-14. https://doi.org/10.1016/j.thromres.2020.04.024

3. Helms J, Tacquard C, Severac F et al (2020) High risk of thrombosis in patients with severe SARS-CoV-2 infection: a multicenter prospective cohort study. Intensive Care Med 46:1089-1098. https://doi.org/10.1007/s00134-020-06062-x
4. Ackermann M, Verleden SE, Kuehnel M et al (2020) Pulmonary vascular endothelialitis, thrombosis, and angiogenesis in Covid19. N Engl J Med 383:120-128. https://doi.org/10.1056/nejmo a2015432

5. Loo J, Spittle DA, Newnham M (2021) COVID-19, immunothrombosis and venous thromboembolism: biological mechanisms. Thorax 76:412-420. https://doi.org/10.1136/thora xjnl-2020-216243

6. Grasselli G, Tonetti T, Protti A et al (2020) Pathophysiology of COVID-19-associated acute respiratory distress syndrome: a multicentre prospective observational study. Lancet Respir Med 8:1201-1208. https://doi.org/10.1016/S2213-2600(20)30370-2

7. Patel BV, Arachchillage DJ, Ridge CA et al (2020) Pulmonary angiopathy in severe COVID-19: physiologic, imaging, and hematologic observations. Am J Respir Crit Care Med 202:690-699. https://doi.org/10.1164/rccm.202004-1412OC

8. Li Q, Huang XT, Li CH et al (2021) CT features of coronavirus disease 2019 (COVID-19) with an emphasis on the vascular enlargement pattern. Eur J Radiol. https://doi.org/10.1016/j.ejrad. 2020.109442

9. Lv H, Chen T, Pan Y et al (2020) Pulmonary vascular enlargement on thoracic $\mathrm{CT}$ for diagnosis and differential diagnosis of COVID-19: a systematic review and meta-analysis. Ann Transl Med 8:878-878. https://doi.org/10.21037/atm-20-4955

10. Bai HX, Hsieh B, Xiong $Z$ et al (2020) Performance of radiologists in differentiating COVID-19 from non-COVID-19 viral pneumonia at chest CT. Radiology 296:E46-E54. https://doi.org/ 10.1148/radiol.2020200823

11. Caruso D, Zerunian M, Polici M et al (2020) Chest CT features of COVID-19 in Rome, Italy. Radiology 296:E79-E85. https://doi. org/10.1148/radiol.2020201237

12. Zhou S, Wang Y, Zhu T, Xia L (2020) CT features of coronavirus disease 2019 (COVID-19) pneumonia in 62 patients in Wuhan, China. Am J Roentgenol 214:1287-1294. https://doi.org/10.2214/ AJR.20.22975

13. Liu J, Zheng X, Tong Q et al (2020) Overlapping and discrete aspects of the pathology and pathogenesis of the emerging human pathogenic coronaviruses SARS-CoV, MERS-CoV, and 2019nCoV. J Med Virol 92:491-494. https://doi.org/10.1002/jmv. 25709

14. Eddy RL, Sin DD (2020) Computed tomography vascular tree-inbud: a novel prognostic imaging biomarker in COVID-19? Am J Respir Crit Care Med 202:642-644. https://doi.org/10.1164/rccm. 202007-2833ED

15. Scaramuzzo G, Gamberini L, Tonetti T et al (2021) Sustained oxygenation improvement after first prone positioning is associated with liberation from mechanical ventilation and mortality in critically ill COVID-19 patients: a cohort study. Ann Intensive Care 11:1-10. https://doi.org/10.1186/s13613-021-00853-1

16. Gamberini L, Tonetti T, Spadaro S, et al (2020) Factors influencing liberation from mechanical ventilation in coronavirus disease 2019: multicenter observational study in fifteen Italian ICUs. $4: 1-12$

17. Tack D, Nollevaux MC, Gevenois PA (2001) Tree-in-bud pattern in neoplastic pulmonary emboli. Am J Roentgenol 176:14211422. https://doi.org/10.2214/ajr.176.6.1761421

18. Lang M, Som A, Carey D et al (2020) Pulmonary vascular manifestations of COVID-19 pneumonia. Radiol Cardiothorac Imaging 2:e200277. https://doi.org/10.1148/ryct.2020200277

19. Hansell DM, Bankier AA, Mcloud TC et al (2008) Fleischner society: glossary of terms for thoracic imaging. Radiology 246:697

20. Yehya N, Harhay MO, Curley MAQ et al (2019) Reappraisal of ventilator-free days in critical care research. Am J Respir Crit Care Med 200:828-836. https://doi.org/10.1164/rccm.201810-2050CP 
21. Ranieri VM, Rubenfeld GD, Thompson BT et al (2012) Acute respiratory distress syndrome: the Berlin definition. JAMA J Am Med Assoc 307:2526-2533. https://doi.org/10.1001/jama.2012. 5669

22. Huang H, Cai S, Li Y et al (2020) Prognostic factors for COVID19 pneumonia progression to severe symptoms based on earlier clinical features: a retrospective analysis. Front Med 7:1-7. https:// doi.org/10.3389/fmed.2020.557453

23. Şanli DET, Yildirim D (2021) A new imaging sign in COVID-19 pneumonia: vascular changes and their correlation with clinical severity of the disease. Diagn Interv Radiol 27:172-180. https:// doi.org/10.5152/dir.2020.20346

24. Herold T, Jurinovic V, Arnreich C et al (2020) Elevated levels of IL- 6 and CRP predict the need for mechanical ventilation in COVID-19. J Allergy Clin Immunol 146:128-136.e4. https://doi. org/10.1016/j.jaci.2020.05.008

25. Esposito A, Palmisano A, Toselli M et al (2021) Chest CT-derived pulmonary artery enlargement at the admission predicts overall survival in COVID-19 patients: insight from 1461 consecutive patients in Italy. Eur Radiol 31:4031-4041. https://doi.org/10. 1007/s00330-020-07622-x

26. Zhu QQ, Gong T, Huang GQ et al (2021) Pulmonary artery trunk enlargement on admission as a predictor of mortality in in-hospital patients with COVID-19. Jpn J Radiol 39:589-597. https://doi. org/10.1007/s11604-021-01094-9

27. Malpani Dhoot N, Goenka U, Ghosh S et al (2020) Assigning computed tomography involvement score in COVID-19 patients: prognosis prediction and impact on management. BJR Open 2:20200024. https://doi.org/10.1259/bjro.20200024

28. Carvalho ARS, Guimarães A, Werberich GM et al (2020) COVID19 chest computed tomography to stratify severity and disease extension by artificial neural network computer-aided diagnosis. Front Med 7:1-11. https://doi.org/10.3389/fmed.2020.577609
29. Ruch Y, Kaeuffer C, Ohana M et al (2020) CT lung lesions as predictors of early death or ICU admission in COVID-19 patients. Clin Microbiol Infect 26:1417.e5-1417.e8. https://doi.org/10. 1016/j.cmi.2020.07.030

30. Levi M, Coppens M (2021) Vascular mechanisms and manifestations of COVID-19. Lancet Respir Med 9:551-553. https://doi. org/10.1016/s2213-2600(21)00221-6

31. Mokhtari T, Hassani F, Ghaffari N et al (2020) COVID-19 and multiorgan failure: a narrative review on potential mechanisms. J Mol Histol 51:613-628. https://doi.org/10.1007/ s10735-020-09915-3

32. Stephens JR, Stümpfle R, Patel P et al (2020) Analysis of critical care severity of illness scoring systems in patients with coronavirus disease 2019: a retrospective analysis of three UK ICUs. Crit Care Med 49:E105-E107

33. Bardi T, Pintado V, Gomez-Rojo M et al (2021) Nosocomial infections associated to COVID-19 in the intensive care unit: clinical characteristics and outcome. Eur J Clin Microbiol Infect Dis 40:495-502. https://doi.org/10.1007/s10096-020-04142-w

34. Ippolito M, Misseri G, Catalisano G et al (2021) Ventilator-associated pneumonia in patients with covid-19: a systematic review and meta-analysis. Antibiotics 10:1-19. https://doi.org/10.3390/ antibiotics 10050545

35. Potere N, Valeriani E, Candeloro M et al (2020) Acute complications and mortality in hospitalized patients with coronavirus disease 2019: a systematic review and meta-analysis. Crit Care 24:1-12. https://doi.org/10.1186/s13054-020-03022-1

Publisher's Note Springer Nature remains neutral with regard to jurisdictional claims in published maps and institutional affiliations.

\section{Authors and Affiliations}

\section{Giorgia Dalpiaz ${ }^{1}$ - Lorenzo Gamberini ${ }^{2}\left(\mathbb{D}\right.$. Aldo Carnevale ${ }^{3}$. Savino Spadaro ${ }^{4}$. Carlo Alberto Mazzoli ${ }^{2}$. Sara Piciucchi ${ }^{5}$. Davide Allegri ${ }^{6}$. Chiara Capozzi ${ }^{7}$. Ersenad Neziri ${ }^{8}$. Maurizio Bartolucci ${ }^{9}$. Francesco Muratore ${ }^{10}$. Francesca Coppola ${ }^{11}$. Antonio Poerio ${ }^{12}$. Emanuela Giampalma ${ }^{13}$ - Luca Baldini ${ }^{14}$. Tommaso Tonetti $^{15}$. Iacopo Cappellini ${ }^{16}$. Davide Colombo ${ }^{17,18}$. Gianluca Zani ${ }^{19} \cdot$ Lorenzo Mellini $^{20} \cdot$ Vanni Agnoletti $^{21}$. Federica Damiani ${ }^{22}$. Giovanni Gordini ${ }^{2}$. Cristiana Laici ${ }^{23}$. Giuliano Gola ${ }^{24}$. Antonella Potalivo ${ }^{25}$. Jonathan Montomoli ${ }^{26}$. Vito Marco Ranieri ${ }^{15}$. Emanuele Russo ${ }^{21}$. Stefania Taddei ${ }^{27}$. Carlo Alberto Volta ${ }^{4}$. Gaetano Scaramuzzo ${ }^{4}$}

1 Department of Radiology, Bellaria Hospital, Bologna, Italy

2 Department of Anaesthesia, Intensive Care and Prehospital Emergency, Ospedale Maggiore Carlo Alberto Pizzardi, Bologna, Italy

3 Department of Radiology, Azienda Ospedaliero-Universitaria S. Anna, Via Aldo Moro, 8, 44121 Cona, Ferrara, Italy

4 Department of Morphology, Surgery and Experimental Medicine, Section of Anaesthesia and Intensive Care, University of Ferrara, Azienda Ospedaliero-Universitaria S. Anna, Via Aldo Moro, 8, 44121 Cona, Ferrara, Italy

5 Department of Radiology, G. B. Morgagni Hospital, Forlì, Italy

6 Department of Clinical Governance and Quality, Bologna Local Healthcare Authority, Bologna, Italy
7 IRCCS Azienda Ospedaliero-Universitaria Di Bologna, Bologna, Italy

8 Radiology Department, SS. Trinità Hospital, ASL Novara, Borgomanero, Italy

9 Radiology Department, Azienda USL Toscana Centro, Prato, Italy

10 Radiology Department, Infermi Hospital, Rimini, Italy

11 Department of Radiology, IRCCS Azienda Ospedaliero-Universitaria di Bologna, Via Albertoni 15, 40138 Bologna, Italy

12 Department of Radiology, Imola Hospital, Imola, Italy

13 Radiology Department, M. Bufalini Hospital, Cesena, Italy

14 Department of Radiology, University Hospital of Modena, Via del Pozzo 71, 41100 Modena, Italy 
15 Alma Mater Studiorum, Dipartimento di Scienze Mediche e Chirurgiche, Anesthesia and Intensive Care Medicine, Policlinico di Sant'Orsola, Università di Bologna, Bologna, Italy

16 Department of Critical Care Section of Anesthesiology and Intensive Care, Azienda USL Toscana Centro, Prato, Italy

17 Traslational Medicine Department, Eastern Piedmont University, Novara, Italy

18 Anesthesiology Department, SS. Trinità Hospital, ASL Novara, Borgomanero, Italy

19 Department of Anesthesia and Intensive Care, Santa Maria Delle Croci Hospital, Ravenna, Italy

20 Department of Radiology, Santa Maria Delle Croci Hospital, Ravenna, Italy

21 Anaesthesia and Intensive Care Unit, M. Bufalini Hospital, Cesena, Italy
22 Department of Anaesthesia, Intensive Care and Pain Therapy, Imola Hospital, Imola, Italy

23 Division of Anesthesiology, Hospital S. Orsola Malpighi, Alma Mater Studiorum University of Bologna, Bologna, Italy

24 Department of Radiology, Azienda Ospedaliera SS. Antonio e Biagio e Cesare Arrigo, Alessandria, Italy

25 Department of Anaesthesia and Intensive Care, Ospedale degli Infermi, Faenza, Italy

26 Department of Anaesthesia and Intensive Care, Infermi Hospital, Rimini, Italy

27 Anaesthesia and Intensive Care Unit, Bentivoglio Hospital, Bentivoglio, Italy 